\title{
Changes in antioxidant status associated with haemodialysis in chronic kidney dis- ease
}

\author{
Adeyemi Ogunleye ${ }^{1}$, Akinwumi A Akinbodewa ${ }^{2}$, Oluseyi A Adejumo ${ }^{2}$, Tosin T Oluwafemi ${ }^{3}$ and \\ Damilola A. Akinfaderin ${ }^{2}$ \\ Ghana Med J 2018; 52(1): 29-33 DOI: http://dx.doi.org/10.4314/gmj.v52i1.6
}

\begin{abstract}
${ }^{1}$ Department of Chemical Pathology, Kidney Care Centre, Ondo, Ondo State, Nigeria ${ }^{2}$ Department of Medicine, University of Medical Science, Ondo, Ondo State, Nigeria ${ }^{3}$ Department of Medical Microbiology, Kidney Care Centre, Ondo, Ondo State, Nigeria
\end{abstract}

Corresponding author: Ogunleye Adeyemi $\quad$ E-mail: yemwum@yahoo.com

Conflict of interest: None declared

\section{SUMMARY}

Oxidative stress has been implicated in the pathogenesis, progression of chronic kidney disease (CKD) and development of cardiovascular complications. Hemodialysis (HD) has also been described to contribute significantly to oxidative stress in CKD patients, though reports are conflicting.

Objective: We evaluated the effects of one session of $\mathrm{HD}$ on the antioxidant capacity and lipid peroxidation in CKD patients.

Method: Thirty-six CKD patients requiring HD were recruited into this study. Participants were naïve to HD and each completed a session of three hours using polysulfone membrane dialyzers. Blood samples were collected before and after dialysis. Total antioxidant capacity (TAC) was measured by ferric reducing antioxidant power (FRAP) while malondialdehyde (MDA) was measured using thiobarbituric acid-reactive substance (TBARS). Comparison was made between pre-HD and post-HD values of TAC and MDA respectively, $p$ value of $<0.05$ was taken as significant.

Result: Mean age and estimated glomerular filtration rate of subjects were $45 \pm 15$ years and $6.3 \pm 4.7 \mathrm{mls} / 1.73 \mathrm{~m}^{2}$ respectively. There was significant decrease in the mean TAC from $1232.2 \pm 495.6 \mu$ mol Trolox equiv/ to $832.4 \pm$ $325.7 \mu$ mol Trolox equiv/L post-dialysis $(\mathrm{p}<0.001)$ while MDA values were similar before and after HD $(11.8 \pm 1.8$ vs $11.8 \pm 2.331) \mu \mathrm{mol} / \mathrm{L}(\mathrm{p}>0.05)$. There was no significant association between changes in antioxidant status following HD with blood flow rate, ultrafiltration volume nor dialyzer per size.

Conclusion: A session of HD in patients with CKD is associated with significant reduction of the total antioxidants capacity; and no effect on MDA levels.

Funding: No external funding received

Keywords: oxidative stress, antioxidants capacity, hemodialysis, chronic kidney disease

\section{INTRODUCTION}

Oxidative stress has been described to play an important role in disease progression and development of cardiovascular complications in chronic kidney disease (CKD) patients. CKD is characterized by an imbalance between pro-oxidant and anti-oxidant factors in favor of oxidants which predisposes them to increased risk of cardiovascular disease and increased mortality. ${ }^{1}$

Though oxidative stress may be directly associated with renal insufficiency as observed in early stages of CKD, it is most pronounced in patients on dialysis. ${ }^{1}$ A number of mechanisms have been postulated as being responsible for oxidative stress in these subjects; certain studies suggested an alteration of the oxidative stress phenomenon, by production of reactive oxygen species on the surface of dialysis membranes and activation of polymorphonuclear leukocytes in patients undergoing hemodialysis (HD) ${ }^{2,3}$, another study suggested loss of antioxidant during the course of HD through the dialyzer membranes ${ }^{4}$, while some studies claim uremia is generally associated with increased oxidative stress. ${ }^{5}$

Haemodialysis, the commonest renal replacement therapy in developing countries, has been reported to induce repetitive bouts of oxidative stress primarily through membrane bio-incompatibility. ${ }^{6,7}$ While CKD is a pro-oxidant state, HD may contribute significantly to oxidative stress in these patients. 
Total antioxidant capacity is described as the sum total of all endogenous and exogenous antioxidant in a medium. ${ }^{8}$ It consist of enzymes such as glutathione peroxidase, catalase, superoxide dismutase as well as macromolecules such as cerulosplasmin, uric acid, ferritin and a number of micromolcules such as ascorbic acid, alpha tocopherol and beta carotene. Combination of these components provides a better protection against reactive species than individual antioxidant.

Malondiadehyde (MDA) is an intermediate product of lipid peroxidation which serves as evidence of oxidation of lipids following HD. Elevated levels of MDA has been reported in CKD as evidence of free radical generation. ${ }^{9}$ This study was designed to evaluate the effects of one session of HD using the standard polysulfone membrane on antioxidant status of newly-diagnosed hemodialysisnaive CKD patients who required $\mathrm{HD}$.

\section{METHODS}

This study was conducted at the Kidney Care Centre, University of Medical Science, Ondo city between April 2015 and March 2016. Ethical approval was obtained from the ethics and research committee, Kidney Care Centre Ondo (under the auspices of Ondo state Hospital Management Board Ondo State, Nigeria.) and all participants gave written consent to participate in the study. Inclusion criteria were newly diagnosed adult Stage 5 CKD patients who required HD and signed written consent to participate in the study. Exclusion criteria were those on antioxidant supplement and lipid lowering medications. $5 \mathrm{ml}$ of venous blood collected just before dialysis (as pre dialysis sample) and 30 minutes after completion of the first session of HD (as post dialysis). Samples were separated and kept at $-2^{\circ} \mathrm{C}$ till analysis. Glomerular filtration rate (GFR) was calculated using the MDRD formula which has been previously validated in Nigerian subjects. ${ }^{10}$

\section{Assay}

Serum creatinine and urea were analyzed using standard spectrophotometric methods (using reagents commercially prepared by RANDOX).

Total antioxidant potential was analyzed using commercially prepared reagent using the ferric reducing antioxidant power (FRAP) assay method as described by Benzie and Strain. ${ }^{11} 50 \mathrm{uL}$ of sample was reacted with $1.5 \mathrm{~mL}$ freshly constituted working reagent (2, 4, 6-tripyridyl-striazine and Ferric chloride hexahydrate) at $37^{\circ} \mathrm{C}$ and result expressed in $\mu \mathrm{mol}$ Trolox equiv/L. MDA was measured using thiobarbituric acid-reactive substance (TBARS) as described by Varshnsey and Kale. ${ }^{12}$
$100 \mathrm{uL}$ of deproteinized sample was added to $1 \mathrm{~mL}$ of TBARS reagents and incubated at $100^{\circ} \mathrm{C}$ for $20 \mathrm{~min}$ expressed in $\mathrm{umol} / \mathrm{L}$.

\section{Statistical analysis.}

Comparison between the mean values of TAC and MDA before and after HD was done using students' t-test. $\mathrm{p}<0.05$ was considered statistically significant in all comparisons.

\section{RESULTS}

A total of $36 \mathrm{CKD}$ patients requiring HD participated in this study, 29 were males and 7 were females. The mean age was $45 \pm 15$ years, systolic blood pressure, diastolic blood pressure, packed cell volume, serum urea, creatinine and estimated GFR before HD were $148.9 \pm$ $29.2 \mathrm{mmHg}, 86.4 \pm 20.0 \mathrm{mmHg}, 21.8 \pm 5.03 \%, 40.7 \pm 19.1$ $\mathrm{mmol} / \mathrm{L}, 1420 \pm 856.0 \mu \mathrm{mol} / \mathrm{L}, 6.3 \pm 4.7 \mathrm{~mL} / \mathrm{min} / 1.73 \mathrm{~m}^{2}$ respectively. Ultrafiltration volume and blood flow rate of the study participants were $1.69 \pm 1.59 \mathrm{~L}$ and $302 \pm$ $60.9 \mathrm{ml} / \mathrm{min}$ respectively (Table 1 ).

Aetiology of CKD among the study participants were hypertension in 12(33.3\%), chronic glomerulonephritis in $11(30.6 \%)$, diabetes mellitus in $7(19.4 \%)$ and others in 6 (16.7\%) (Table 2).

Table1 Characteristics of study population.

\begin{tabular}{|l|l|}
\hline Parameters & Mean \pm SD $/ \mathbf{~ n ( \% ) ~}$ \\
\hline Age & $45 \pm 15$ years \\
\hline Systolic blood pressure & $148.9 \pm 29.2 \mathrm{mmHg}$ \\
\hline Diastolic blood pressure & $86.4 \pm 20.0 \mathrm{mmHg}$ \\
\hline Packed cell volume & $21.8 \pm 5.03 \%$ \\
\hline Serum urea & $40.7 \pm 19.1 \mathrm{mmol} / \mathrm{L}$ \\
\hline Serum creatinine & $1420 \pm 856.0 \mathrm{micromol} / \mathrm{L}$ \\
\hline Estimated GFR & $6.3 \pm 4.7 \mathrm{~mL} / \mathrm{min} / 1.73 \mathrm{~m}^{2}$ \\
\hline Ultrafiltration volume & $1.69 \pm 1.59 \mathrm{~L}$ \\
\hline Blood flow rate & $302 \pm 60.9 \mathrm{ml} / \mathrm{min}$ \\
\hline
\end{tabular}

Table 2 Aetiology of CKD among the participants.

\begin{tabular}{|l|l|}
\hline Aetiology of CKD & $\mathbf{n ( \% )}$ \\
\hline Hypertension & $12(32.4 \%)$, \\
\hline Chronic glomerulonephritis & $11(29.7 \%)$ \\
\hline Diabetes mellitus & $7(18.9)$ \\
\hline Others & $6(16.2 \%)$. \\
\hline
\end{tabular}

There was significant decrease in the mean TAC from $1232.2 \pm 495.6$ to $832.4 \pm 325.7 \mu \mathrm{mol}$ Trolox equiv/L $(\mathrm{p}<0.001)$ after a single session of HD. There was no significant change in mean MDA values after a single HD session $(11.8 \pm 1.8$ vs $11.8 \pm 2.331 \mu \mathrm{mol} / \mathrm{L}(\mathrm{p}>0.05))(\mathrm{Ta}-$ ble 3). 
Blood flow rate, ultrafiltration volume and blood pressure did not significantly affect changes in MDA and TAC following HD ( $>0.05)$ (Tables 4 and 5).

Table 3 The effect of a single session of Haemodialysis on MDA and TAC

\begin{tabular}{|l|l|l|l|}
\hline Parameter & Pre-dialysis & Post-dialysis & p value \\
\hline TAC & $1232.2 \pm 495.6$ & $832.4 \pm 325.7$ & $<0.001$ \\
\hline MDA & $11.8 \pm 1.8$ & $11.8 \pm 2.331$ & 0.753 \\
\hline
\end{tabular}

Table 4 Association between changes in MDA and ultrafiltration volume, blood flow rate and blood pressure

\begin{tabular}{|l|l|l|}
\hline & p-value & $\mathbf{R}$ \\
\hline Ultrafiltration volume & 0.73 & -0.07 \\
\hline Blood Flow Rate & 0.75 & -0.06 \\
\hline Systolic Blood Pressure & 0.50 & 0.12 \\
\hline Diastolic Blood Pressure & 0.43 & -0.14 \\
\hline
\end{tabular}

Table 5 Association between changes in TAC and ultrafiltration volume, blood flow rate and blood pressure

\begin{tabular}{|l|l|l|}
\hline & p-value & R \\
\hline Ultrafiltration volume & 0.73 & -0.04 \\
\hline Blood Flow Rate & 0.53 & -0.11 \\
\hline Systolic Blood Pressure & 0.89 & -0.24 \\
\hline Diastolic Blood Pressure & 0.23 & 0.24 \\
\hline
\end{tabular}

\section{DISCUSSION}

Antioxidants are important in patients with CKD to retard disease progression, and reduce the risk of premature development of cardiovascular disease (CVD) and death. Total antioxidant capacity is a measure of overall body defence against free radicals, it is made up of enzymatic and non-enzymatic components.

This study showed a significant reduction in the total antioxidant capacity in patients undergoing HD. Oxidative stress (OS) has been implicated in the pathogenesis of cardiovascular death and CKD patients are at increased risk of both OS and cardiovascular death. Incidence of cardiovascular events and death has been reported to increase soon after commencement of $\mathrm{HD}$, however the mechanism responsible is not fully understood. ${ }^{13}$ This study further suggests that loss of antioxidants across the semipermeable dialyzer bio-incompatible membrane during the course of HD may play a significant role in increased oxidative stress in patients undergoing $\mathrm{HD}$ and may partly contribute to increased mortality. This study is in consonance with observed significant difference in the levels of total antioxidants after HD in previous studies. ${ }^{4,14}$
Significant imbalance in the amount of pro-oxidants and antioxidants have been shown to be prevalent among CKD patients, while this has been associated with uremia and production of inflammatory cells such as polymorphonuclear cells (PMNs), loss of antioxidants during dialysis procedure plays a significant role. Oxidative stress in HD patients is associated with extracorporeal treatments ${ }^{15}$, repeated contact of blood cells with bio-incompatible membrane causing leukocyte activation and consequent increased formation of ROS, pro-inflammatory cytokine and pro-oxidants. ${ }^{16}$ The inflammatory cells triggers increased secretion of myeloperoxidase and nicotinamide adenine dinucleotide phosphate oxidase that will also increase the production of ROS. Leakage of blood antioxidants through the dialysis membrane and higher consumption of antioxidant during HD has been described. ${ }^{17}$

In this study, the levels of plasma MDA, which serves as evidence of lipid peroxidation were similar before and after HD. Plasma MDA is an intermediate product of lipid peroxidation and a marker of oxidative stress. The result of our study suggests that HD may not be associated with the removal of pro-oxidants molecules such as MDA in CKD patients. Though elevated levels of MDA has been demonstrated in patients with varying degrees of renal functions ${ }^{9,18}$, this study showed that a session of HD did not yield a significant reduction in its plasma levels in CKD patients undergoing first session of HD. This is in agreement with earlier study ${ }^{19}$ who observed a non-significant difference following HD though it's not stated whether participants were incipient or have been on dialysis. In another study by Biasioli et $a l^{20}$, post dialysis sample was collected 30 minutes after completing HD, MDA values were reported to be similar to those pre-dialysis. That study however recruited participants who were on regular HD.

In contrast to some studies that observed significant increase in lipid peroxidation following HD evidenced by elevated MDA level post-dialysis ${ }^{4,17,21}$, our study did not show increased lipid peroxidation rather it did show inability of the HD to remove MDA (pro-oxidant molecule). The difference may be associated with duration of dialysis and method of selection of participants. Our study considered incipient dialysis patients and a duration of three hours while most other studies didn't state the duration of HD, some studies also considered patients on regular maintenance HD. ${ }^{11,21}$ The difference may also be associated with the possibility of serum MDA value attaining equilibrium after $\mathrm{HD}$ as this study sampled post-dialysis 30 minutes after completing HD while others collected immediately after HD. 
This is similar to finding in a study that sampled post dialysis 30 minutes after completing $\mathrm{HD}^{20}$ suggesting that time of collection may be associated with the difference.

This study showed that blood flow rate, ultrafiltration volume and blood pressure at the onset of dialysis did not significantly affect changes in TAC and MDA following HD. However, there are no previous reports to compare with our findings.

Our study confirmed significant decrease in total antioxidant capacity associated with HD that has been documented in previous studies and further suggests that the possible mechanism may be associated with loss of soluble components during HD. Possible cumulative effects of this coupled with non-clearance of MDA pose dialysis patients at increased risk of oxidative stress. This may enhance disease progression, cardiovascular complications and increase mortality among these patients. However, the use of vitamin E coated dialyzers has been suggested to reduce oxidative stress and endothelial dysfunction associated with HD. ${ }^{22}$

\section{CONCLUSION}

This study has further shown that HD is associated with significant alteration in antioxidant status of CKD patients. Even a single session of HD may contribute to OS in CKD patients through loss of antioxidants across dialyzer bio incompatible membrane. Clinical trials may be necessary to ascertain the probable beneficial effects of antioxidants supplements and antioxidant-coated dialyzers.

\section{REFERENCES}

1. Francesco Locatelli, Bernard Canaud, Kai-Uwe Eckardt, Peter Stenvinkel, Christoph Wanner and Carmine Zoccali. Oxidative stress in end-stage renal disease: an emerging threat to patient outcome. Nephrol. Dial. Transplant. 2003; 18 (7): 1272-1280.

2. Fiorillo C, Oliviero C, Rizzuti G, Nediani C, Pacini A, Nassi P. Oxidative stress and antioxidant defenses in renal patients receiving regular haemodialysis. Clin Chem Lab Med. 1998; 36(3):149-53.

3. Descamps-Latscha B, Drüeke T, Witko-Sarsat V. Dialysis-induced oxidative stress: biological aspects, clinical consequences, and therapy. Semin Dial 2001; 14:193-199

4. Ajala MO, Ogunro P.S, Alli Odun. Effect of hemodialysis on total antioxidant status of chronic renal failure patients in government hospitals in Lagos Nigeria. Niger J Clin Pract 2011:14 (2):154-158.

5. Witko-Sarsat V, Friedlander M., Capeillère-Blandin C., Nguyen-KhoaAnh T., Thu NguyenJohanna,
ZingraffPaul Jungers, Descamps-Latscha B. Advanced oxidation protein products as a novel marker of oxidative stress in uremia. Kidney International May 1996; Vol. 49(5):1304-1313.

6. Kuo K.-L. and D.-C. Tarng, "Oxidative stress in chronic kidney disease". Adaptive Medicine, 2010; 2( 2): 87-94,.

7. Descamps-Latscha B, Goldfarb B, Nguyen AT., Kazatchkine M. Establishing the relationship between complement activation and stimulation of phagocyte oxidative metabolism in hemodialyzed patients: a randomized prospective study. Nephron 1991; 59(2):279-285

8. Rezaie A, Parker R.D, Abdollahi M. oxidative stress and pathogenesis of inflammatory bowel disease: An epiphenomenon or the cause? Digestive Diseases and Sciences. 2007: 52 (9):2015-2021.

9. Ong-Ajyooth L, Ong-Ajyooth S, Sirisalee K, Nilwarangkur S. Lipoproteins and lipid peroxidation abnormalities in patients with chronic renal disease. JMedAssoc. Thai. 1996; 79:505-12.

10. Abefe SA, Abiola AF, Olubunmi AA, Adewale A. Utility of predicted creatinine clearance using MDRD formula compared with other predictive formulas in Nigerian patients. Saudi J Kidney Dis Transpl. 2009; 20:86-90.

11. Benzie IF, Strain JJ. The ferric reducing ability of plasma (FRAP) as a measure of "antioxidant power": the FRAP assay. Anal Biochem. 1996; 239(1):70-6.

12. Varshney R, Kale RF. Effect of Calmodulin antagonists on radiation induced lipid peroxidation in Microsomes. Int. J. Rad. Biol., 1990; 58: 733- 743.

13. Bruce Robinson, Jinyao Zhang, Hal Morgenstern, Brian D. Bradbury, Leslie J. Ng, Keith McCullough, Brenda Gillespie, Raymond Hakim, Hugh Rayner, Joan Fort, Tadao Akizawa, Francesca Tentori, and Ronald Pisoni. World-wide, mortality is a high risk soon after initiation of hemodialysis. Kidney Int. 2014; 85(1): 158-165.

14. Kadkhodaee, M., Hemmati, M., Zahmatkesh, M., Ghaznavi, R., Mirershadi, F., Mahdavi-Mazde, M. and Seifi, B. Assessment of Plasma Antioxidant Status in Hemodialysis Patients. Therapeutic Apheresis and Dialysis. 2008; 12: 147-151.

15. Kobayashi S, Moriya H, Aso K, Ohtake T. Vitamin E-bonded hemodialyzer improves atherosclerosis associated with a rheo- logical improvement of circulating red blood cells. Kidney Int. 2003; 63(5):1881-1887.

16. Nguyen AT, Lethias C, Zingraff J, Herbelin A, Naret C, Descamps-Latscha B. Hemodialysis membraneinduced activation of phagocyte oxidative metabolism detected in vivo and invitro with microamounts of whole blood. Kidney Int 1985; 28:158-67. 
17. Mehryar Zargari, Omid Sedighi Influence of Hemodialysis on Lipid Peroxidation, Enzymatic and Non- Enzymatic Antioxidant Capacity in Chronic Renal Failure Patients. Nephro Urol 2015; 7(4): 28526.

18. Kachhawa K, Varma M, Kachhawa P, Agrawal D, Shaikh M, Kumar S. Study of dyslipidemia and antioxidant status in chronic kidney disease patients at a hospital in South East Asia. J Health Res Rev 2016; (3):28-30.

19. Lin TH, Chen JG, Liaw JM, Juang JG. Trace elements and lipid peroxidation in uremic patients on hemodialysis. Biol Trace Elem Res. 1996; 51(3):277- 83.
20. Biasioli S, Schiavon R, Petrosino L, Cavallini L, Cavalcanti G, De Fanti E, Zambello A, Borin D: Role of cellulosic and non- cellulosic membranes in hyperhomocysteinemia and oxidative stress. ASAIO J. 2000; 46: 625-634,.

21. Ogunro P S, Oluyombo R, Ajala M O, Oshodi T. T. The effect of a membrane dialyzer during hemodialysis on the antioxidant status and lipid peroxidation of patients with end-stage renal disease. Saudi J Kidney Dis Transpl. 2014; 25:1186-93

22. Jing Huang, Bin Yi, Ai-mei Li \& Hao Zhang. Effects of vitamin E-coated dialysis membranes on anemia, nutrition and dyslipidemia status in hemodialysis patients: a meta-analysis, Renal Failure. 2015;37 (3): $398-407$ 